\title{
Silica triflate as an efficient catalyst for the solvent-free synthesis of 3,4-dihydropyrimidin-2(1H)-ones
}

\author{
Farhad Shirini, *a Katayoun Marjani, ${ }^{* b}$ and Hossein Taherpour Nahzomi ${ }^{\text {a,b }}$ \\ ${ }^{a}$ Department of Chemistry, College of Science, Guilan University, Rasht, 41335, Iran, \\ Fax: +981313220066 \\ ${ }^{b}$ Faculty of Chemistry, Teacher Training University, Tehran, 15614, Iran \\ E-mail:shirini@guilan.ac.ir
}

\begin{abstract}
Silica triflate was prepared as a stable white powder by the reaction of silica gel with trifluoromethylsulfonyl chloride at room temperature. This reagent efficiently catalyzed the onepot synthesis of 3,4-dihydropyrimidin-2-(1H)-ones and -thiones (DHPMs) under solvent-free reaction conditions in good to high yields.
\end{abstract}

Keywords: Aldehydes, Biginelli condensation, 3,4-dihydropyrimidin-2-(1H)-ones, solvent-free reaction conditions, silica triflate

\section{Introduction}

The considerable interest in 3,4-dihydropyrimidin-2(1H)-one- type products results from their structural similarity to dihydropyridines (DHP), a class of compounds showing remarkable pharmacological properties as calcium channel antagonists and which are extensively used as therapeutics in the clinical treatment of cardiovascular diseases such as hypertension, cardiac arrhythmias, or angina pectoris. ${ }^{1}$ Dihydropyrimidinone derivatives (DHPMs) show similar biological activity. ${ }^{2}$

The Biginelli reaction is constituted by a one-pot acid-catalyzed condensation of a $\beta$ ketoester and a urea leading to 3,4-dihydropyrimidin-2-(1H)-one (DHPM). ${ }^{3}$ Synthesis of 3,4dihydropyrimidin-2-(1H)-ones and -thiones (DHPMs) have became the center of increasing attention using both solution and solid-phase reaction techniques in recent years. ${ }^{4,5}$ Improved procedures with different types of catalysts ${ }^{6-8}$ and conditions ${ }^{9}$ have been reported with the aim of overcoming the main drawback of the Biginelli reaction, which is represented by the modest yields. However, some of the reported methods also suffer from drawbacks such as cumbersome product isolation procedures and environmental pollution. ${ }^{6,10-15}$ Moreover some of the methods are only practical for aromatic aldehydes. Therefore, a need still exists for versatile, simple and 
environmentally friendly processes whereby DHPMs may be formed under milder and practicable conditions.

In recent years, the interest of our research group has focused on the development of applications of silica gel and its new derivatives in organic chemistry. In the course of our studies, we have reported that these reagents can be used efficiently in many organic reactions. ${ }^{17-}$ 20 In continuation of these studies we have found that silica gel reacts with trifluoromethylsulfonyl chloride to give silica triflate (I) as a white powder. It is interesting to note that the reaction is easy and clean. Because of the immediate evolution of $\mathrm{HCl}$ gas from the reaction vessel, the method does not need any work-up procedure (Scheme 1).

We hoped that silica triflate, as a new silica based reagent, might be able to accelerate many organic reactions, as do the other reported triflates. Herein, we wish to report the abilities of this reagent for the one-pot synthesis of 3,4-dihydropyrimidin-2-(1H)-ones and -thiones in high yields (Scheme 2).

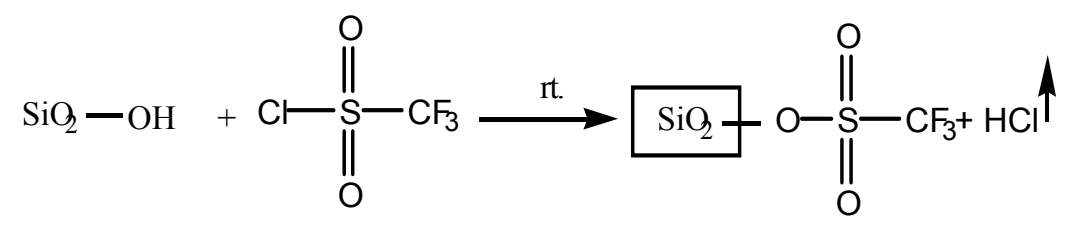

\section{Scheme 1}

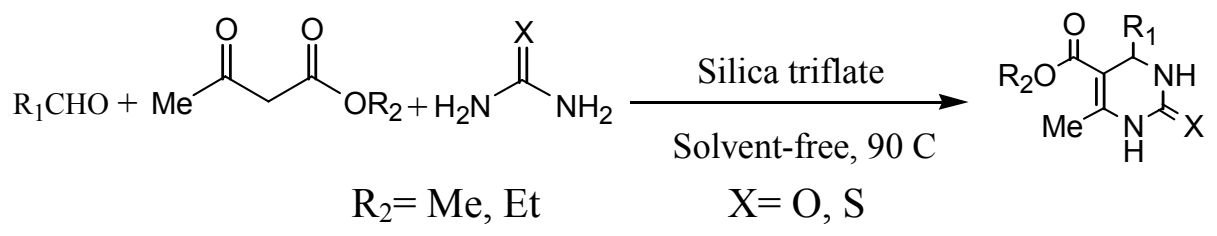

\section{Scheme 2}

Optimization of the reaction conditions is carried out by the condensation of benzaldehyde, ethyl acetoacetate and urea under solvent-free conditions. The best result was achieved by carrying out the reaction of benzaldehyde, ethyl acetoacetate and urea (with 1:1:1.5 mol ratio) in the presence of $0.03 \mathrm{~g}$ of silica triflate at $90^{\circ} \mathrm{C}$ for $5 \mathrm{~min}$ under solvent-free conditions (Table 1 , entry 1).

Aliphatic and several aromatic aldehydes carrying either electron-releasing or electronwithdrawing substituents were also afforded high yields of the products (Table 1). Methyl acetoacetate and thiourea were also used with similar success, to provide the corresponding 3,4dihydropyrimidin-2-( $1 H)$-thiones. ${ }^{21}$

We have found that silica triflate is a reusable catalyst and, even after five runs, the catalytic activity of the reagent was almost the same as that of the freshly used catalyst. The same IR 
spectra are used for the demonstration of the stability of the composition of the reagent before and after the reactions.

A plausible mechanism for the synthesis of 3,4-dihydropyrimidin-2-(1H)-ones and -thiones using this method is shown in Scheme 3 , based on the literature, ${ }^{22}$ our own observations and the obtained results.

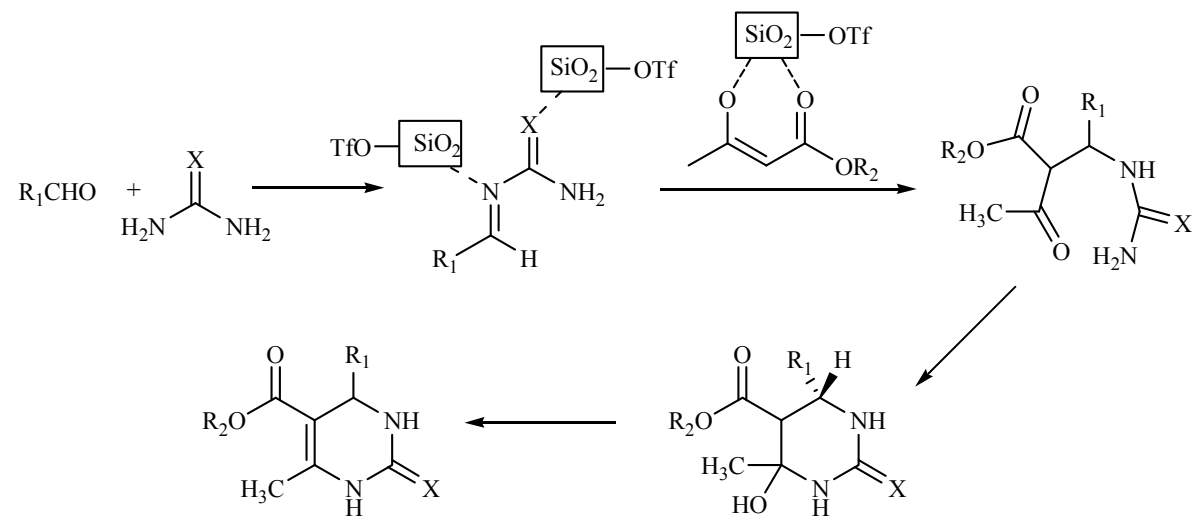

\section{Scheme 3}

In order to show the efficiency of the present method, Table 2 compares some of the results with some of those reported in the literature using silica-based reagents. ${ }^{23-25}$

In conclusion, the present procedure demonstrates the preparation of a new triflate derivative based on silica gel and its application in the solvent-free synthesis of 3,4-dihydropyrimidin2(1H)-ones and -thiones. Based on our studies, this method offers several advantages including mild reaction conditions, good to high yields of the products, shorter reaction times, facile purification, solvent-free reaction conditions and a simple experimental procedure. 
Table 1. Solvent-free synthesis of 3,4-dihydropyrimidin-2(1H)-ones and -thiones in the presence of silica triflate ${ }^{\mathrm{a}}$

\begin{tabular}{ccccccc}
\hline Entry & $\mathrm{R}_{1}$ & $\mathrm{R}_{2}$ & $\mathrm{X}$ & Yield $\%{ }^{\mathrm{b}}$ & $\mathrm{mp}\left({ }^{0} \mathrm{C}\right)$ & Lit. mp ${ }^{0} \mathrm{C}$, and ref. \\
\hline 1 & $\mathrm{C}_{6} \mathrm{H}_{5}$ & $\mathrm{Et}$ & $\mathrm{O}$ & 95 & $204-206$ & $202-204^{7}$ \\
2 & $4-\mathrm{ClC}_{6} \mathrm{H}_{4}$ & $\mathrm{Et}$ & $\mathrm{O}$ & 92 & $208-210$ & $213-215^{7}$ \\
3 & $3-\mathrm{NO}_{2} \mathrm{C}_{6} \mathrm{H}_{4}$ & $\mathrm{Et}$ & $\mathrm{O}$ & 85 & $229-230$ & $226-227^{7}$ \\
4 & $2-\mathrm{NO}_{2} \mathrm{C}_{6} \mathrm{H}_{4}$ & $\mathrm{Et}$ & $\mathrm{O}$ & 70 & $205-207$ & $206-208^{7}$ \\
5 & $4-\mathrm{MeOC}_{6} \mathrm{H}_{4}$ & $\mathrm{Et}$ & $\mathrm{O}$ & 82 & $198-200$ & $201-203^{7}$ \\
6 & $2-\mathrm{MeOC}_{6} \mathrm{H}_{4}$ & $\mathrm{Et}$ & $\mathrm{O}$ & 70 & $260-262$ & $262^{7}$ \\
7 & $4-\mathrm{NMe}_{2} \mathrm{C}_{6} \mathrm{H}_{4}$ & $\mathrm{Et}$ & $\mathrm{O}$ & 80 & $257-258$ & $256-257^{26}$ \\
8 & $\mathrm{C}_{6} \mathrm{H}_{5} \mathrm{CH}=\mathrm{CH}$ & $\mathrm{Et}$ & $\mathrm{O}$ & 70 & $231-232$ & $232-235^{7}$ \\
9 & $2-$ furyl & $\mathrm{Et}$ & $\mathrm{O}$ & 95 & $207-209$ & $209-211^{27}$ \\
10 & $\mathrm{n}-\mathrm{Bu}$ & $\mathrm{Et}$ & $\mathrm{O}$ & 85 & $156-158$ & $157-158^{12}$ \\
11 & $\mathrm{C}_{6} \mathrm{H}_{5}$ & $\mathrm{Et}$ & $\mathrm{S}$ & 95 & $208-209$ & $208-210^{7}$ \\
12 & $4-\mathrm{ClC}_{6} \mathrm{H}_{4}$ & $\mathrm{Et}$ & $\mathrm{S}$ & 85 & $191-192$ & $192-195^{26}$ \\
13 & $4-\mathrm{MeOC}_{6} \mathrm{H}_{4}$ & $\mathrm{Et}$ & $\mathrm{S}$ & 87 & $153-156$ & $150-152^{7}$ \\
14 & $3-\mathrm{NO}_{2} \mathrm{C}_{6} \mathrm{H}_{4}$ & $\mathrm{Et}$ & $\mathrm{S}$ & 70 & $203-205$ & $206-207^{7}$ \\
15 & $\mathrm{C}_{6} \mathrm{H}_{5}$ & $\mathrm{Me}$ & $\mathrm{O}$ & 85 & $229-231$ & $233-236^{7}$ \\
16 & $4-\mathrm{Cl}_{6} \mathrm{C}_{6} \mathrm{H}_{4}$ & $\mathrm{Me}$ & $\mathrm{O}$ & 85 & $203-205$ & $204-207^{7}$ \\
17 & $4-\mathrm{MeOC}_{6} \mathrm{H}_{4}$ & $\mathrm{Me}$ & $\mathrm{O}$ & 70 & $189-192$ & $192-194^{7}$ \\
18 & $2-$ furyl $^{7}$ & $\mathrm{Me}$ & $\mathrm{O}$ & 95 & $208-209$ & $210-212^{6}$ \\
\hline
\end{tabular}

${ }^{a}$ Products were characterized by their physical constants, comparison with authentic samples, and IR and NMR spectroscopy. ${ }^{\mathrm{b}}$ Isolated yield.

Table 2. Comparison of some of the results obtained by our method (1), with some of those reported for $\mathrm{KAl}\left(\mathrm{SO}_{4}\right)_{2} \cdot 12 \mathrm{H}_{2} \mathrm{O}$-supported silica gel (2), ${ }^{23}$ sulfonic acid covalently anchored onto silica (3) ${ }^{24}$ and silica-supported heteropoly acids (4) ${ }^{25}$

\begin{tabular}{cccccc}
\hline \multirow{2}{*}{ Entry } & Product & \multicolumn{4}{c}{ Time (h)/ Yield (\%) } \\
\cline { 3 - 6 } & & $(1)$ & $(2)$ & $(3)$ & $(4)$ \\
\cline { 3 - 6 } 1 & Table 1, entry 1 & $5(\mathrm{~min}) / 95$ & $4 / 92$ & $8 / 90$ & $1 / 95$ \\
2 & Table 1, entry 5 & $5(\mathrm{~min}) / 82$ & $4 / 91$ & $7 / 92$ & $1 / 95$ \\
3 & Table 1, entry 12 & $5(\mathrm{~min}) / 85$ & - & $8 / 95$ & $1.1 / 87$ \\
4 & Table 1, entry 15 & $5(\mathrm{~min}) / 85$ & $4 / 91$ & - & $1 / 92$ \\
\hline
\end{tabular}




\section{Experimental Section}

General Procedures. Chemicals were purchased from Fluka, Merck and Aldrich Chemical Companies. Yields refer to isolated products. Products were characterized by their physical constants, comparison with authentic samples and IR and NMR spectroscopy. The purity determination of the substrates and reaction monitoring were accompanied by TLC on silica gel PolyGram SILG/ UV 254 plates.

Preparation of silica triflate. A $500 \mathrm{~mL}$ suction flask, charged with $18.0 \mathrm{~g}$ silica gel (type 60 , $15-40 \mu \mathrm{m})$, was equipped with constant pressure dropping funnel containing trifluoromethanesulfonyl chloride $(8.4 \mathrm{~g}, 0.05 \mathrm{~mol})$ and gas inlet tube for conducting $\mathrm{HCl}$ gas over water as an adsorbing liquid. Trifluoromethanesulfonyl chloride was added dropwise over a period of $30 \mathrm{~min}$ and stirred slowly at room temperature for $30 \mathrm{~min}$. The mixture was then heated to $60^{\circ} \mathrm{C}$ while it was stirring for $1 \mathrm{~h}$ to remove all $\mathrm{HCl}$ and the excess amounts of trifluoromethanesulfonyl chloride. The reaction mixture was washed with $50 \mathrm{~mL}$ of dry $\mathrm{CH}_{2} \mathrm{Cl}_{2}$ and dried under vacuum. Silica triflate was obtained as a white solid (20.38-20.62 g) which was stored in a capped bottle; IR (KBr): 1255, 1230, 1130, 1010, 955, 820, 650, 530, $500 \mathrm{~cm}^{-1}$.

\section{General experimental procedure}

A mixture of $\beta$-dicarbonyl compound ( $1 \mathrm{mmol})$, aldehyde $(1 \mathrm{mmol})$, urea or thiourea $(1.5 \mathrm{mmol})$ and silica triflate $(0.03 \mathrm{~g})$ was heated in an oil bath $\left(90^{\circ} \mathrm{C}\right)$ under stirring for $5 \mathrm{~min}$. After completion (monitored by TLC), $\mathrm{CH}_{3} \mathrm{CN}(5 \mathrm{~mL}$ ) was added, the reagent was separated by filtration and the solvent was evaporated. The mixture was poured into water $(5 \mathrm{~mL})$. The precipitate was filtered and successively washed with water $(5 \mathrm{~mL})$ and petroleum ether-EtOAc $(5: 1,5 \mathrm{~mL})$. The crude product was purified by recrystallization to give the pure products in good to high yields.

\section{Selected physical data for compounds}

Table 1, entry 1. M.p. $204-206{ }^{\circ} \mathrm{C} .{ }^{1} \mathrm{H}-\mathrm{NMR}\left(\mathrm{DMSO}_{-} \mathrm{d}_{6}\right): \delta: 1.06$ (t, 3H, J=6.84, $\left.\mathrm{CH}_{3}\right), 2.24(\mathrm{~s}$, $\left.3 \mathrm{H}, \mathrm{CH}_{3}\right), 3.94\left(\mathrm{q}, 2 \mathrm{H}, \mathrm{J}=6.75, \mathrm{CH}_{2}\right), 5.13(\mathrm{~d}, 1 \mathrm{H}, \mathrm{J}=3.06, \mathrm{H}-4), 7.21-7.37(\mathrm{~m}, 5 \mathrm{H}, \mathrm{ArH}), 7.73$ (brs, 1H, NH), 9.19 (brs, 1H, NH). IR (KBr): $v=3245,3118,2987,1725,1701,1649 \mathrm{~cm}^{-1}$.

Table 1, entry 5. M.p. $198-200{ }^{\circ} \mathrm{C} .1 \mathrm{H}-\mathrm{NMR}$ (DMSO-d6): $\delta: 1.10$ (t, 3H, J= 7.08, $\mathrm{CH}_{3}$ ), 2.17 (s, $\left.3 \mathrm{H}, \mathrm{CH}_{3}\right), 3.71$ (s, 3H, CH3), 3.96 (q, 2H, J= 7.14, CH2), 5.07 (d, 1H, J=2.97, H-7), 6.85 (d, 2H, $\mathrm{J}=8.49, \mathrm{ArH}), 7.12(\mathrm{~d}, 2 \mathrm{H}, \mathrm{J}=8.55, \mathrm{ArH}), 7.67$ (brs, $1 \mathrm{H}, \mathrm{NH}), 9.15$ (brs, $1 \mathrm{H}, \mathrm{NH}) . \mathrm{IR}(\mathrm{KBr}): \mathrm{v}=$ $3436,3247,3113,2929,1724,1705,1649 \mathrm{~cm}^{-1}$.

Table 1, entry 9. M.p. $207-209^{\circ} \mathrm{C} .1 \mathrm{H}-\mathrm{NMR}\left(\mathrm{DMSO}_{-} \mathrm{d}_{6}\right.$ ): $\delta 1.05$ (t, 3H, J= 7.08, CH3), 2.22 (s, $\left.3 \mathrm{H}, \mathrm{CH}_{3}\right), 3.98$ (q, 2H, J=6.99, $\left.\mathrm{CH}_{2}\right), 5.19(\mathrm{~d}, 1 \mathrm{H}, \mathrm{J}=3.0, \mathrm{H}-4), 6.08$ (d, 1H, J= 2.94, ArH), 6.35 (s, 1H, ArH). 7.55 (s, 1H, ArH), 7.76 (brs, 1H, NH), 9.25 (brs, 1H, NH). IR (KBr): v=3342, $3242,3117,2985,1700,1645 \mathrm{~cm}^{-1}$. 
Table 1, entry 13. M.p. $153-156^{\circ} \mathrm{C} .1 \mathrm{H}-\mathrm{NMR}\left(\mathrm{DMSO}_{\mathrm{d}}\right)$ ): $\delta: 1.08\left(\mathrm{t}, 3 \mathrm{H}, \mathrm{J}=6.93, \mathrm{CH}_{3}\right), 2.28$ (s, $\left.3 \mathrm{H}, \mathrm{CH}_{3}\right), 3.72\left(\mathrm{~s}, 3 \mathrm{H}, \mathrm{CH}_{3}\right), 3.96$ (q, $\left.2 \mathrm{H}, \mathrm{J}=7.17, \mathrm{CH}_{2}\right), 5.10$ (d, $\left.1 \mathrm{H}, \mathrm{J}=3.6, \mathrm{H}-4\right), 6.87$ (d, $2 \mathrm{H}, \mathrm{J}=8.64, \mathrm{ArH}$ ), 7.09 (d, 2H, J= 8.64, ArH), 9.60 (brs, 1H, NH), 10.28 (brs, 1H, NH). IR (KBr): $v=3445,3315,3175,2965,1667,1617,1575,1510 \mathrm{~cm}^{-1}$.

\section{Acknowledgements}

Partial support of this work by the Guilan and Teacher Training Universities Research Councils is acknowledged. F. Shirini is thankful to the Guilan Science and Technology Park (GSTP) for its continuous support. F. Shirini is also indebted to Dr. M. Mottaghitalab for his assistance.

\section{References}

1. (a) Jauk, B.; Belaj, F.; Kappe, C. O. J. Chem. Soc., Perkin Trans 1 1999, 307. (b) Rovynak, G. C.; Kimball, S. C.; Beyer, B.; Cucinotta, G.; Dimarco, J. D.; Gougoutas, J.; Hedberg, A.; Malley, M.; McCarthy, J. P.; Zhang, R.; Moreland, S. J. Med. Chem. 1995, 38, 119. (c) Atwal, K. S.; Rovnyak, G. C.; Kimball, S. D.; Floyd, D. M.; Moreland, S.; Swanson, B. N.; Gougoutas, J. Z.; Schwarts, J.; Smillie, K. M.; Malley, M. F. J. Med. Chem. 1990, 33, 2629.

2. Atwal, K. S.; Swanson, B. N.; Unger, S. E.; Floyd, D. M.; Moreland, S.; Hedberg, A.; O'Reilly, B. C. J. Med. Chem. 1991, 34, 806.

3. Biginelli, P. Gazz. Chim. Ital. 1893, 23, 360.

4. Kappe, C. O. Acc. Chem. Res. 2000, 33, 879.

5. (a) Kappe, C. O. Med. Chem. Lett. 2000, 10, 49. (b) Kappe, C. O.; Falsone, S. F.; Fabian, W. M. F.; Belaj, F. Heterocycles 1999, 51, 77. (c) McDonald, A. L.; Overman, L. E. J. Org. Chem. 1999, 64, 1520. (d) Yadav, J. S.; Kumar, S. P.; Kondaji, G.; Rao, R. S.; Nagaiah, K. Chem. Lett. 2004, 33, 1168. (e) Yadav, J. S.; Raddy, B. V. S.; Sridhar, P.; Reddy, J. S. S.; Nagaiah, K.; Lingaiah, N.; Saiprasad, P. S. Eur. J. Org. Chem. 2004, 552. (f) Yadav, J. S.; Raddy, B. V. S.; Naidu, J. J.; Sadashiv, K. Chem. Lett. 2004, 33, 926.

6. Ranu, B. C.; Hajra, A.; Jana, U. J. Org. Chem. 2000, 65, 6270.

7. Salehi, P.; Dabiri, M.; Zolfigol, M. A.; Bodaghi Fard, M. A. Tetrahedron Lett. 2003, 44, 2889.

8. Dondoni, A.; Massi, A.; Sabbatini, S. Tetrahedron Lett. 2001, 42, 4495.

9. Stadler, A.; Kappe, C. O. J. Chem. Soc., Perkin Trans. 2 2000, 1363.

10. Hu, E. H.; Sidler, D.R.; Dolling, U. H. J. Org. Chem. 1998, 63, 3454.

11. Yadav, J. S.; Subba, B. V.; Reddy, K. B.; Raj, K. S.; Prasad, A. R. J. Chem. Soc., Perkin Trans. 1 2001, 1939.

12. Ma, Y.; Qian, C.; Wang, L.; Yang, M. J. Org. Chem. 2000, 65, 3864.

13. Lu, J.; Bai, Y.; Wang, Z.; Yang, B.; Ma, H. Tetrahedron Lett. 2000, 41, 9075. 
14. Fu, N. Y.; Yuan, Y. F.; Cao, Z.; Wang, S. W.; Wang, J. T.; Peppe, C. Tetrahedron 2002, 58, 4801.

15. Kumar, K. A.; Kasthuraiah, M.; Reddy, C. S.; Reddy, C. D. Tetrahedron Lett. 2001, 42, 7873.

16. Kappe, C. O.; Kumar, D.; Varma, R. S. Synthesis 1999, 1799.

17. Zolfigol, M. A.; Shirini, F.; Mohammadpoor-Baltork, I.; Ghorbani-Choghamarani, A.; Hajjami, M.; Sedaghat, A. M. Mendeleev Commun. 2005, 113.

18. Shirini, F.; Zolfigol, M. A.; Mohammadi, K. Bull. Korean Chem. Soc. 2004, 25, 325.

19. Shirini, F.; Zolfigol, M. A.; Mohammadi, K. Phosphorus, Sulfur, and Silicon and the Related Elements 2003, 178, 2357.

20. Shirini, F.; Zolfiogl, M. A.; Khaleghi, M.; Mohammadpoor-Baltork, I. Synth. Commun. 2003, 33, 1839.

21. Dondoni, A.; Massi, A.; Sabbatini, S. Tetrahedron Lett. 2002, 43, 5913.

22. Su, W.; Li, J.; Zheng, Z.; Shen, Y. Tetrahedron Lett. 2005, 46, 6037.

23. Azizian, J.; Mohammadi, A. A.; Karimi, A. R.; Mohammadizadeh, M. R. Applied Chem. A: General 2006, 300, 85.

24. Gupta, R.; Paul, S.; Gupta, R. J. Mol. Catal. A: Chem. 2006, 266, 50.

25. Rafiee, E.; Shahbazi, F. J. Mol. Catal. A.: Chem. 2006, 250, 57.

26. Lu, J.; Bai, Y. J. Synthesis 2002, 466.

27. Paraskar, A. S.; DewKar, G. K.; Sudalai A. Tetrahedron Lett. 2003, 44, 3305. 